An application of Theorem 1 now yields (15).

\title{
BIBLIOGRAPHY
}

1. Stefan Bergman, The kernel function and conformal mapping, Mathematical Surveys, no. 5, New York, American Mathematical Society, 1950.

HARVARD UNIVERSITY

\section{REMARK ON THE HURWITZ ZETA FUNCTION}

T. M. APOSTOL

The Hurwitz zeta function, defined for $0<a \leqq 1, \Re(s)>1$, by

$$
\zeta(s, a)=\sum_{n=0}^{\infty} \frac{1}{(a+n)^{s}},
$$

is given [2] ${ }^{1}$ in the negative half-plane by means of

$$
\begin{aligned}
\zeta(1-s, a)= & \frac{2 \Gamma(s)}{(2 \pi)^{s}}\left(\cos \frac{\pi s}{2} \sum_{n=1}^{\infty} \frac{\cos (2 \pi a n)}{n^{s}}\right. \\
& \left.+\sin \frac{\pi s}{2} \sum_{n=1}^{\infty} \frac{\sin (2 \pi a n)}{n^{s}}\right) \\
= & \frac{2 \Gamma(s)}{(2 \pi)^{s}} \sum_{n=1}^{\infty} \frac{\cos (\pi s / 2-2 \pi a n)}{n^{s}} \quad(\Re(s)>1) .
\end{aligned}
$$

The functional equation for the Riemann zeta function is obtained from (2) upon setting $a=1$.

The more general function

$$
\phi(x, a, s)=\sum_{n=0}^{\infty} \frac{e^{2 \pi i n x}}{(a+n)^{s}}
$$

reduces to $\zeta(s, a)$ when $x$ is an integer. Lerch [1] derived the transformation formula

$$
\begin{aligned}
\phi(x, a, 1-s)=\frac{\Gamma(s)}{(2 \pi)^{\circ}}\left\{e^{\pi i(s / 2-2 a x)} \phi(-a, x, s)\right. \\
\left.+e^{\pi i(-s / 2+2 a(1-x))} \phi(a, 1-x, s)\right\}
\end{aligned}
$$

Presented to the Society, June 16, 1951; received by the editors August 24, 1950 and, in revised form, October 9, 1950.

${ }^{1}$ Numbers in brackets refer to the bibliography at the end of the paper. 
this equation being valid for $0<x<1,0<a \leqq 1$.

It is the purpose of this note to show how (2) can be derived by using (4). The method becomes obvious upon noting that Hurwitz functions can also be obtained from Lerch functions by considering the sum

$$
\begin{aligned}
\sum_{t=1}^{k-1} \phi\left(\frac{t}{k}, a, s\right)=\sum_{n=0}^{\infty}(a+n)^{-s} \sum_{t=1}^{k-1} e^{2 \pi i n t / k} & \\
= & (k-1) \sum_{n=0, n \equiv 0(\bmod k)}^{\infty}(a+n)^{-s}-\sum_{n=0, n \neq 0(\bmod k)}^{\infty}(a+n)^{-s} \\
& =k^{1-s} \sum_{n=0}^{\infty}(a / k+n)^{-s}-\sum_{n=0}^{\infty}(a+n)^{-s} \\
& =k^{1-s} \zeta(s, a / k)-\zeta(s, a) .
\end{aligned}
$$

The above rearrangements are all valid if $\Re(s)>1$ and the final result holds for all $s$ by analytic continuation. Replacing $s$ by $1-s$ we have

$$
\sum_{t=1}^{k-1} \phi\left(\frac{t}{k}, a, 1-s\right)=k^{s} \zeta\left(1-s, \frac{a}{k}\right)-\zeta(1-s, a) .
$$

Now we write $x=t / k$ in the right member of (4), assume $\Re(s)>1$, and sum on $t$ to obtain

$$
\begin{aligned}
\sum_{t=1}^{k-1} \phi\left(\frac{t}{k}, a, 1-s\right)=\frac{\Gamma(s)}{(2 \pi)^{s}}\left\{\sum_{t=1}^{k-1} \sum_{n=0}^{\infty} \frac{e^{\pi i(s / 2-2 a n-2 a t / k)}}{(t / k+n)^{*}}\right. \\
\left.+\sum_{t=1}^{k-1} \sum_{n=0}^{\infty} \frac{e^{\pi i(-8 / 2+2 a n+2 a(1-t / k))}}{(1-t / k+n)^{s}}\right\} .
\end{aligned}
$$

Replacing $1-t / k$ by $t / k$ in the second term in the braces does not alter the sum over $t$ and we obtain

$$
\sum_{t=1}^{k-1} \phi\left(\frac{t}{k}, a, 1-s\right)=\frac{2 \Gamma(s)}{(2 \pi)^{s}} \sum_{t=1}^{k-1} \sum_{n=0}^{\infty} \frac{\cos (\pi s / 2-2 \pi a n-2 \pi a t / k)}{(t / k+n)^{s}} .
$$

If we write $\lambda=n k+t$, then $\lambda$ takes on all positive integer values which are not multiples of $k$ as $n, t$ run through their respective ranges, and our sum becomes

$$
\begin{aligned}
\sum_{t=1}^{k-1} \phi\left(\frac{t}{k},\right. & a, 1-s) \\
& =\frac{2 \Gamma(s) k^{s}}{(2 \pi)^{s}} \sum_{\lambda=1}^{\infty}\left(\frac{\cos (\pi s / 2-2 \pi a \lambda / k)}{\lambda^{s}}-\frac{\cos (\pi s / 2-2 \pi a \lambda)}{(k \lambda)^{s}}\right) .
\end{aligned}
$$


Comparison with (5) yields the equation

$$
k^{*} \Delta(s, a / k)=\Delta(s, a),
$$

where

$$
\Delta(s, a)=\zeta(1-s, a)-\frac{2 \Gamma(s)}{(2 \pi)^{s}} \sum_{n=1}^{\infty} \frac{\cos (\pi s / 2-2 \pi a n)}{n^{2}} .
$$

We wish to show that $\Delta(s, a)$ vanishes identically for $\Re(s)>1$. To do this we keep $s$ fixed and real and consider $\Delta(s, a)$ for $a \rightarrow 0+$. For $s>1$, equation (1) shows that we have

$$
\lim _{a \rightarrow 0+}\left(\zeta(s, a)-a^{-s}\right)=\zeta(s) .
$$

Using partial integration in (1) we obtain, for $s>0$,

$$
\zeta(s, a)=a^{-s}+\frac{a^{1-s}}{s-1}-s \sum_{n=0}^{\infty} \int_{0}^{1} \frac{u}{(n+a+u)^{s+1}} d u,
$$

from which we find that $(8)$ holds for $0<s<1$. Using partial integration in (9) we obtain, for $s>-1$,

$$
\begin{aligned}
\zeta(s, a)=a^{-s} & +\frac{a^{1-s}}{s-1}-\frac{s}{2 !}\left(\zeta(s+1, a)-a^{-s-1}\right) \\
& -\frac{s(s+1)}{2 !} \sum_{n=0}^{\infty} \int_{0}^{1} \frac{u^{2}}{(n+a+u)^{s+2}} d u .
\end{aligned}
$$

We then find, by (9) and (10),

$$
\lim _{a \rightarrow 0+} \zeta(s, a)=\zeta(s) \quad(-1<s<0) .
$$

If we now let $a \rightarrow 0+$ in equation (7) we find

$$
\lim _{a \rightarrow 0+} \Delta(s, a)=\zeta(1-s)-\frac{2 \Gamma(s)}{(2 \pi)^{s}} \cos \frac{\pi s}{2} \zeta(s),
$$

this being valid for $1<s<2$. But if we let $k \rightarrow \infty$ in (6), keeping $1<s<2$, we see by (11) that we have

$$
\zeta(1-s)=\frac{2 \Gamma(s)}{(2 \pi)^{s}} \cos \frac{\pi s}{2} \zeta(s),
$$

or $\lim _{a \rightarrow 0+} \Delta(s, a)=\Delta(s, 1)=0$. Now we put $a=1$ in (6) to obtain

$$
\Delta(s, 1 / k)=0 \quad(k=1,2,3, \cdots) .
$$


Now consider any rational $p / q, 0<p<q$. Take $a=1-p / q, k=q-p$ in (6). We obtain

$$
(q-p)^{s} \Delta(s, 1 / q)=\Delta(s, 1-p / q)
$$

and hence, by (12),

$$
\Delta(s, 1-p / q)=0
$$

Hence $\Delta(s, a)$ vanishes at all rational $a$ in $0<a<1$. Since $\Delta(s, a)$ is continuous in $a$ in this interval, $\Delta(s, a)=0$ for every $a, 0<a<1$. Hence $\Delta(s, a)$ vanishes identically for $1<s<2$, and by analytic continuation for $\Re(s)>1$.

\section{BIBLIOGRAPHY}

1. M. Lerch, Note sur la fonction $\Re(w, x, s)=\sum_{k=0}^{\infty} e^{2 k \pi i s} /(w+k)^{\circ}$, Acta Math. vol. 11 (1887) pp. 19-24.

2. E. T. Whittaker and G. N. Watson, Modern analysis, Cambridge, 1945, p. 269.

California Institute of Technology 